\title{
Herramientas clínicas para el diagnóstico de miastenia gravis
}

\section{Objetivo}

Determinar que datos del interrogatorio y el examen físico permiten identificar a aquellos pacientes con diagnóstico de miastenia gravis.

\section{Diseño}

Revisión sistemática.

\section{Fuentes de datos y selección de estudios}

Se realizó una búsqueda en MEDLINE (enero 1966-enero 2005) limitándose la misma a artículos en inglés. Se utilizaron los términos "myastenia gravis, diagnosis and test". Se revisó la bibliografía de los artículos encontrados. Se seleccionaron aquellos estudios en los que se evaluaba la presencia de algún síntoma o signo en pacientes con y sin la presencia de miastenia gravis. De 640 artículos encontrados, se seleccionaron 33 y de estos, 15 cumplieron los criterios de inclusión. Dos autores evaluaron los estudios en forma independiente para determinar la calidad de los mismos.

\section{Resultados}

La presencia de "habla progresivamente mas incomprensible a medida que se prolonga la duración del discurso" y la presencia del signo de "peek" (separación involuntaria leve de los párpados y aparición de la esclerótica posterior al cierre de los mismos) aumentaron la probabilidad diagnóstica de miastenia gravis (CPP 4,5 IC $95 \%$ 1,2-17 y CPP 30 IC 95\% 3,2-278) respectivamente. La ausencia de los mismos no disminuyó significativamente la presencia de miastenia.

Los estudios identificados evaluaron solo dos hallazgos más del examen físico o la historia clínica ("persistencia de comida en la boca después de tragar" y "movimientos repentinos de los ojos") y ni la presencia o ausencia de estos modificó la probabilidad diagnóstica de miastenia.

En relación a maniobras simples se encontró que la prueba de hielo (apoyar hielo sobre el ojo que presenta ptosis durante dos minutos y posteriormente evaluar una apertura palpebral mayor a $2 \mathrm{~mm}$ ) de ser positiva, aumenta la probabilidad de miastenia (CPP 24 IC95\% 8,5-67) y si fuera negativa disminuye la probabilidad (CPN 0,16 IC95\% 0,09-0,27).

Una respuesta positiva a una medicación anticolinesterasa incrementa la probabilidad de miastenia (CPP 15 IC95\% 7,5-31) y la ausencia de respuesta la disminuye (CPN 0,11 IC95\% 0,06-0,21). Una respuesta anormal en la prueba de sueño (no lograr una apertura palpebral mayor a $2 \mathrm{~mm}$ después de permanecer en una habitación en penumbras descansando durante al menos 20 minutos) aumenta la probabilidad diagnóstica de miastenia (CPP 53 IC95\% 3,4-832) y un resultado normal de dicha prueba (lograr una apertura palpebral mayor a $2 \mathrm{~mm}$ ) descarta la presencia de la misma (CPN 0,01 IC95\% 0-0,16).

\section{Conclusiones}

Algunos hallazgos del interrogatorio y examen físico combinados con maniobras simples que se pueden realizar en el consultorio (prueba del hielo, del sueño, anticolinesterasa) permiten mejorar la identificación de aquellos pacientes con miastenia. Estos resultados deben interpretarse con precaución debido a las diferencias entre la prevalencia en la población general y la reportada en los estudios evaluados.

Fuente de financiamiento: no reportada

\section{Comentario}

La Miastenia Gravis (MG) Autoinmune es la principal enfermedad que afecta a la placa neuromuscular. Su variada y fluctuante presentación clínica plantea un dilema diagnóstico que generalmente no se devela en la primer visita. Si bien el tiempo promedio que se demora en hacer el diagnostico se redujo de cuatro años a uno en la ultima década; mas allá de los síntomas iniciales que presente, el paciente miasténico suele recorrer varios especialistas hasta conseguir el diagnostico definitivo. Esto se explica por la fluctuación y extensión de los síntomas y signos que provoca esta enfermedad, gran "imitadora" de otras.

El articulo de Scherer y col. plantea una buena alternativa para el diagnostico de MG con pruebas sencillas y realizables en el consultorio. Metodológicamente es correcto y las pruebas diagnósticas del Edrofonio, hielo y reposo - están bien descriptas en artículo original. Si bien estas parecen de fácil realización, resaltamos la importancia del signo clínico que se va a evaluar, generalmente los relacionados a los músculos oculares, para que tenga valor diagnóstico.

El diagnóstico de MG se basa en la clínica, pruebas farmacológicas (edrofonio, neostigmina) pruebas electrofisiológicas (estimulación repetitiva y electromiograma de fibra única) y serológicas (dosaje de anticuerpos ACRA y anti-MuSK); siendo importante en nuestro medio estar alerta a la calidad y la validación de las pruebas electrofisiológicas y serológicas.

Recomendamos a los lectores el artículo de Meriggioli y Sanders ${ }^{1}$ quienes decriben una estrategia racional para el estudio de estos individuos. Recordamos que dentro de los síndromes miasténicos existen cuadro clínicos de presentación poco frecuente como la afectación bulbar pura o la de los músculos distales, fenotipos peculiares como los pacientes con MG generalizada seronegativa con anticuerpos anti-MuSK positivo o MG congénita. También se observan cuadros que por su severidad parecen miopatías en los que la fluctuación clínica es muy difícil de evaluar. Por supuesto que la aproximación diagnóstica de estos "subgrupos", como también del síndrome miasteniforme de Lambert Eaton requiere gran entrenamiento, por lo que se recomienda interconsultar a estos pacientes con el especialista.

\section{Conclusión del comentador}

El diagnostico de MG se basa en las manifestaciones clínicas y los exámenes complementarios: pruebas farmacológicas, electrofisiológicas y serológicas.

Marcelo Rugiero [ Médico Especialista en Neurología. Servicio de Neurología del Hospital Italiano de Buenos Aires, Sección Enfermedades Neuromusculares. Fundación de Ayuda Integral al Miasténico. ]

Rugiero M. Herramientas clínicas para el diagnóstico de miastenia gravis. Evid. actual. práct. ambul. 2006; 9(3) ;78. May-Jun. 2006. Comentado de: Scherer K Bedlack R, Simel D. Does This Patient Have Myasthenia Gravis? JAMA. 2005; 293:1906-1914. PMID: 15840866. 